\title{
Síntomas de la depresión en mujeres de Dubai - Un estudio piloto
}

\author{
Sultana Sulaiman $\mathrm{PhD}^{*}$ \\ Dinesh Bhugra, FRCPsych, PhD** \\ Padmal De Silva FBPsS ${ }^{* * *}$ \\ * Jefatura General de Policía de Dubai, PO \\ BOX 279, Dubai \\ EMIRATOS ÁRABES UNIDOS \\ ** Instituto de Psiquiatría, De Crespigny \\ Park, London SE5 8AF \\ *** Instituto de Psiquiatría, De Crespigny \\ Park, London SE5 8AF \\ REINO UNIDO
}

\begin{abstract}
RESUMEN - Mediante la utilización de criterios y métodos estándar de diagnóstico para investigar tanto la gravedad de la depresión como la naturaleza de los síntomas del trastorno depresivo en pacientes del sexo femenino en Dubai, llevamos a cabo el estudio de 56 casos (con una edad media de 32,3 años) que padecían depresión diagnosticada según criterios ICD-10 mientras recibían atención en el Servicio de Psiquiatría de un hospital de Dubai. Se emplearon como instrumentos de evaluación el BDI y el MMPI-D. Los resultados se evaluaron atendiendo a la edad, estado civil, nivel de estudios y situación laboral, así como a la clase socioeconómica. Las conclusiones del presente estudio piloto indican que cuando acuden a los servicios psiquiátricos las pacientes no alfabetizadas suelen mostrar síntomas de depresión más grave que las pacientes con estudios. Estas pacientes obtuvieron en los tests puntuaciones significativamente superiores en comparación con las pacientes americanas evaluadas según el manual BDI en los aspectos de sentimiento de castigo, pérdida del apetito, pérdida de peso, cambio de aspecto físico, fatiga, obsesión somática, insomnio, irritabilidad y retraimiento social. Mostraron una probabilidad inferior de puntuación en los apartados de autorechazo, ideas de suicidio, indecisión y autoacusación. Estas conclusiones preliminares se analizan en relación con la investigación que actualmente se está llevando a cabo.
\end{abstract}

\section{Introducción}

Durante las dos últimas décadas, aunque ha existido un interés considerable por el estudio de las variaciones interculturales en la epidemiología de la depresión, todavía sabemos muy poco acerca de la naturaleza y la frecuencia de la depresión en las diversas culturas, debido a las limitaciones en los 
métodos de investigación de los que se dispone en la actualidad (Marsella 1987). Existen poderosas razones teóricas que hacen pensar que tanto el grado como la manifestación de la depresión varían significativamente a través de las distintas culturas (Kleinman 1986, Kleinman \& Good 1986, Marsella 1987, Bebbington 1993). Diversos estudios se han centrado en las posibles diferencias que existen en cuanto a la forma en que se manifiestan los síntomas de la dolencia en los distintos entornos culturales (Marsella 1987, Krause 1992). Hasta hace muy poco no ha existido información cuantitativa en los Emiratos Árabes Unidos (EAU) para aportar indicios acerca de si la sintomatología depresiva está presente de forma significativa en su población nativa femenina y en caso de ser así, sobre cuál es su naturaleza y si existen diferencias en la manifestación de la misma entre las mujeres de los EAU y las de otras culturas.

Se han realizado pocos estudios sobre la frecuencia de los síntomas de la depresión entre las mujeres de Dubai, que forma parte de los EAU. Ghubash (1992) se centró en la enfermedad metal entre las mujeres de Dubai y aportó cierta información acerca de la depresión en las mujeres. La investigadora observó que los valores más altos en cuanto a trastorno mental correspondían a la depresión (13\% de la muestra) que era el trastorno mental más común entre las mujeres de Dubai. Ghareeb (1992) realizó un estudio sobre la naturaleza de la depresión en la adolescencia en los EAU y señaló que no se hallaron diferencias por causa de la edad en dicha población, pero que existía una clara diferencia de incidencia por sexos. Las adolescentes presentan unos valores relativos a depresión más elevados que la población adolescente masculina y la proporción de individuos del sexo masculino y del sexo femenino con síntomas de depresión es de 2:3. Estos resultados concuerdan con muchos de los estudios anterior- mente realizados. El índice de depresión entre mujeres y hombres en la mayoría de las sociedades se presenta en una proporción de dos a uno (e.g., Hawton 1993, Ashurst \& Hall 1991, MIND 1992, Kleinman 1986). Parece ser que las circunstancias y el puesto de las mujeres en la sociedad dan lugar a una cierta vulnerabilidad para el padecimiento de depresión (MIND 1992). Las variables sociodemográficas, las experiencias vitales estresantes y un apoyo social insuficiente se han considerado factores que contribuyen a esta mayor incidencia de la depresión, especialmente entre las mujeres (e.g., Mahatme et al. 1989, Bebbington 1987, 1993, Avasthi et al. 1991, Lehtinen \& Joukamaa 1994, Paykel 1994, Olfson \& Klerman 1992, Weissman 1981, Kleinman \& Good 1985). Stoppard (1989) se ha referido a la discriminación social y a una situación socioeconómica desfavorecida de las mujeres en la sociedad como posibles factores clave que subyacen en el desarrollo de una indefensión adquirida y por ello de una mayor propensión a la depresión en las mujeres en comparación con los hombres.

Los Emiratos Árabes Unidos, especialmente Dubai, en un país multicultural en el que las costumbres, hábitos y estructura social varían notablemente entre los miembros de los distintos grupos raciales y étnicos. Por ello, es importante disponer de información cualitativa y cuantitativa acerca de la frecuencia de la depresión entre las mujeres de Dubai y de la naturaleza de los síntomas de dicho trastorno. Es interesante señalar que la transformación sociocultural en la sociedad de los EAU ha sido rápida y generalizada, abarcando todas las facetas esenciales de la vida social. Esta transformación no fue programada sino espontánea y en gran medida sin planificación. El proceso transformador que precisó de dos siglos en las sociedades occidentales tuvo lugar en los EAU en sólo veinte años. Pare- 
ce ser que tales cambios han hecho que la infraestructura de la sociedad y las normas y tradiciones sociales se resientan y que todo ello se manifieste de forma paralela en la proliferación de trastornos psicológicos.

El presente estudio presenta un doble propósito. El primer objetivo es explorar la asociación entre depresión y variables sociodemográficas (edad, estado civil, nivel de estudios y situación laboral, así como clase socioeconómica); y el segundo propósito es evaluar si existen variaciones interculturales en la forma en que se presentan los síntomas de la depresión.

\section{Material y Metodología}

\section{Ámbito y periodo de estudio:}

El estudio se llevó a cabo durante un periodo de nueve meses en el Emirato de Dubai, Emirato que ocupa el segundo lugar en cuanto a tamaño de los Emiratos Árabes Unidos, y que está integrado por siete Emiratos semiautónomos: Abu Dhabi, Dubai, Ras Al Khaima, Umm Al Qaywain, Sharjah, Fujairah y Ajman.

\section{Individuos:}

Cincuenta y seis pacientes del sexo femenino fueron reclutadas de entre las pacientes remitidas de forma consecutiva a los Servicios Externos de Psiquiatría de un hospital general de Dubai. Sólo se incluyeron pacientes cuyo diagnóstico consistía en el padecimiento de depresión clínica de acuerdo con la ICD-10 (WHO 1992). Las pacientes con síntomas psicóticos patentes, síndromes cerebrales orgánicos y retraso mental fueron excluidas del estudio. Ninguna de las pacientes rehusó participar en el estudio.

\section{Medidas:}

En el estudio se emplearon los siguientes cuestionarios:

(a) El Beck Depression Inventory (BDI) que consta de 21 apartados y que había sido traducido al árabe y previamente validado por Ghareeb (1988). Este inventario se diseño en un principio para evaluar la gravedad de la depresión en pacientes con diagnóstico psiquiátrico y abarca síntomas cognitivos (apartados 1-13) y síntomas somáticos (apartados 14-21), (Lustman et al. 1992). Beck et al. (1993) han señalado que una puntuación de 10 en el BDI puede considerarse como el límite de una depresión moderada.

(b) El Minnesota Multiphasic Personality Inventory, Depression Scale (MMPI-D) que previamente había sido traducido al árabe y validado (Al-Haj 1980). Esta escala se desarrolló en principio para evaluar la depresión sintomática. Muchos de los 60 apartados de esta escala hacen referencia a diversos aspectos de la depresión, como la denegación de felicidad y valía personal, el retraso y el retraimiento psicomotor y la falta de interés en el entorno personal. Otros apartados de la escala abarcan una variedad de otros síntomas y comportamientos, incluyendo dolencias físicas, preocupación o tensión, negación de los impulsos, dificultad para controlar los procesos del pensamiento propio y el fervor religioso.

\section{Procedimiento:}

Se abordaron todos los casos con un diagnóstico clínico ICD-10 de depresión (el diagnóstico lo efectuaron psiquiatras locales con experiencia). Se recogieron cincuenta y 
seis casos durante el periodo de estudio. Se obtuvo el consentimiento con pleno conocimiento. Todas las pacientes respondieron los cuestionarios en el propio hospital. Si las pacientes no sabían leer, el primero de los autores les efectuaba su lectura.

\section{Estadística:}

Las diferencias entre las medias del grupo se evaluaron a través del análisis de la varianza (ANOVA) para determinar si las variables de edad, estado civil, nivel de estudios y situación laboral, así como la de clase socioeconómica eran independientes o bien asociadas con respecto a la gravedad de la depresión. Igualmente se utilizaron tablas de contingencia con dos factores de variación (análisis chi-cuadrado) para examinar las diferencias entre los síntomas de depresión en las pacientes del sexo femenino de Dubai y en las pacientes de Estados Unidos, haciendo uso de la información del manual del BDI 1993. Debido a la naturaleza preliminar de este estudio, se consideró la existencia de asociaciación cuando $\mathrm{p}<0,05$ para poder describir posibles tendencias que merezcan una investigación posterior.

\section{Resultados}

Veinticinco pacientes obtuvieron el diagnóstico de un ICD-296 (Depresión Grave) y 31 pacientes fueron diagnosticadas de ICD 311 (Trastorno Depresivo, no clasificado en ningún otro lugar). Del total del grupo, cinco eran pacientes internas y el resto pacientes externas. El rango de edad de las pacientes iba de los 20 a los 40 años, con una media de edad de 32,3 años. Siete (12\%) de los sujetos de estudio estaban entre los 20 y los 24 años, once (20\%) entre los 25 y los 29 años, trece (23\%) entre los 30 y los 34 años y veinticinco (45\%) tenían entre 35 y 40 años.

La mayoría de las mujeres estaban casadas (59\%) o habían estado casadas (el 7\% estaban divorciadas y el $4 \%$ eran viudas) y el $30 \%$ no estaban casadas. Todas las pacientes sujeto de estudio fueron clasificadas como nativas de los Emiratos, es decir, habían nacido y recibido su educación en los Emiratos Árabes Unidos y pertenecían mayoritariamente a la cultura musulmana. Una mayoría tenía la titulación de Educación Secundaria o de Licenciatura universitaria $(64 \%)$ o titulación superior $(4 \%)$. Del total, el $88 \%$ sabía leer y escribir y el $12 \%$ carecía de alfabetización. El cincuenta y nueve por ciento eran amas de casa, el $37 \%$ eran trabajadoras en activo y el $4 \%$ eran estudiantes. La mayoría de las pacientes sujetas a estudio procedían de la clase media $(52 \%)$ y el $21 \%$ y $27 \%$ pertenecían a las clases baja y alta respectivamente.

De las 56 participantes en el estudio que cumplimentaron el BDI, 30 de ellas (54\%) padecían Depresión Grave, 14 (25\%) sufrían Depresión moderada y 12 (21\%) Depresión leve. Según los resultados del MMPID, $13(23 \%)$ padecían una depresión muy acusada, 10 (18\%) depresión acusada, 15 (27\%) depresión moderada, 16 (28\%) depresión modal y 2 (4\%) depresión leve. La puntuación media relativa a depresión según el BDI fue de 27,6 con SD de 11,89. En el MMPI-D la media fue de 30,08 (7,27). Mediante el análisis del coeficiente Pearson, hallamos que el BDI guarda una relación significativa con el MMPI-D ( $\mathrm{r}=0,67, \mathrm{p}<$ 0,001 ) en esta muestra.

La duración media de los trastornos depresivos de las pacientes fue de dos años. Todas las pacientes recibían medicación para sus trastornos depresivos. 
En los análisis que se recogen a continuación, se trataron las respuestas de las pacientes al BDI y al MMPI-D de dos maneras distintas. En primer lugar, atendiendo a un formato cuantitativo, las respuestas al BDI se valoraron numéricamente, por lo que las puntuaciones más altas se consideraron como indicativas de una mayor sintomatología de depresión; también se sumaron las puntuaciones de las pacientes en cada uno de los distintos apartados para obtener una puntuación total del BDI. Para el MMPI-D, las respuestas también se valoraron de acuerdo con la clave de puntuación, por lo que una mayor puntuación representaría una Depresión Mayor. En este tratamiento cuantitativo, el total de las puntuaciones del BDI y del MMPI-D, respectivamente, fueron las magnitudes dependientes, en el análisis de varianza, en el que la edad, el estado civil, el nivel de estudios, la situación laboral, y la clase socioeconómica de las pacientes se consideraron como variables independientes.

En un segundo conjunto de análisis se adoptó un tratamiento más cualitativo, en el que los límites para las puntuaciones totales del BDI y del MMPI-D se consideraron como clínicamente relevantes. Beck et al. (1993) han sugerido que una puntuación total de 10 a 18 en el BDI es indicativa de depresión leve, de 19 a 29 es indicativa de depresión moderada y de 30 a 63 es indicativa de depresión severa. De forma similar, Hathaway y McKinley (1989) han sugerido que la puntuación total de 40 o inferior en el MMPI-D es indicativa de depresión ligera, de 41 a 55 es indicativa de depresión modal, de 56 a 65 es indicativa de depresión moderada, de 66 a 75 es indicativa de depresión acusada y de 76 o más es indicativa de depresión muy acusada. Según este enfoque cualitativo, se clasificó a las pacientes de acuerdo con la gravedad de la depresión y las frecuencias de gravedad de la depresión en las pacientes se calcularon en función de su edad, estado civil, nivel de estudios, situación laboral y clase socioeconómica. Los datos relativos se presentan en la tabla I.

Se realizó el análisis simple de varianza (ANOVA) para determinar si las variables arriba descritas eran independientes o bien asociadas a la gravedad de la depresión.

Atendiendo a las puntuaciones tanto del BDI como del MMPI-D, la edad, el estado civil y la situación laboral no presentaban relación con el grado de depresión.

\section{Nivel de estudios}

En el BDI existían diferencias significativas $(F=3,52, d f=2,53, p=, 037)$ entre la gravedad de la depresión y el nivel de estudios. Las pacientes sin alfabetización presentaban un mayor número de síntomas de depresión que las pacientes de los otros dos grupos alfabetizados.

\section{Clase socioeconómica}

En el BDI existían diferencias significativas $(\mathrm{F}=4,25, \mathrm{df}=2,53, \mathrm{p}=, 019)$ entre la clase socioeconómica y la gravedad de la depresión. Las pacientes pertenecientes a la clase baja presentaban un mayor número de síntomas de depresión que las de las clases media y alta.

Los datos relativos se presentan en la tabla II.

\section{Análisis individual de los apartados del BDI}

También se examinaron por medio de tablas de contingencia con dos factores de 
Tabla I

Nivel de depresión en BDI y MMPI-D y variables socio-demográficas para un total de 56 pacientes que comprenden el total de la muestra

\begin{tabular}{|c|c|c|c|c|c|c|c|c|c|}
\hline \multirow[b]{2}{*}{$\begin{array}{l}\text { Variables } \\
\text { demográficas }\end{array}$} & \multicolumn{4}{|c|}{ BDI } & \multirow[b]{2}{*}{$\begin{array}{l}\text { Baja } \\
\text { Total }\end{array}$} & \multicolumn{4}{|c|}{ MMPI-D } \\
\hline & Mild & Moder & Sever & Total & & Modal & Moder & Alta & V. Alta \\
\hline \multicolumn{10}{|l|}{ Edad } \\
\hline $20-24$ & $3(43)$ & - & $4(57)$ & $7(12)$ & $1(14)$ & $2(29)$ & $1(14)$ & $1(14)$ & $2(29)$ \\
\hline $25-29$ & $1(09)$ & $4(36)$ & $6(55)$ & $11(20)$ & $7(12)$ & & & & \\
\hline $30-34$ & $3(24)$ & $5(38)$ & $5(38)$ & $13(23)$ & $1(09)$ & $3(27)$ & $3(27)$ & $3(27)$ & $1(09)$ \\
\hline \multirow[t]{3}{*}{$35-40$} & $5(20)$ & $5(20)$ & $15(60)$ & $25(45)$ & $11(20)$ & & & & \\
\hline & & & & & $\begin{array}{c}- \\
13(23)\end{array}$ & $7(54)$ & $3(23)$ & $1(08)$ & $2(15)$ \\
\hline & & & & & $\begin{array}{c}- \\
25(45)\end{array}$ & $4(16)$ & $8(32)$ & $5(20)$ & $8(32)$ \\
\hline \multicolumn{10}{|l|}{ Estado civil } \\
\hline Casado/a & $6(18)$ & $7(21)$ & $20(60)$ & $33(59)$ & $1(03)$ & $8(24)$ & $10(30)$ & $6(18)$ & $8(24)$ \\
\hline Soltero/a & $6(35)$ & $5(30)$ & $6(35)$ & $17(30)$ & $33(59)$ & & & & \\
\hline Divorciado/a & - & $2(50)$ & $2(50)$ & $4(07)$ & 1(06) & $7(41)$ & $4(23)$ & $3(18)$ & $2(12)$ \\
\hline \multirow[t]{3}{*}{ Viudo/a } & - & - & $2(100)$ & $2(04)$ & $17(30)$ & & & & \\
\hline & & & & & $\begin{array}{c}- \\
4(07)\end{array}$ & $1(25)$ & $1(25)$ & - & $2(50)$ \\
\hline & & & & & $2(04)$ & - & - & $1(50)$ & $1(50)$ \\
\hline \multicolumn{10}{|l|}{ Educación } \\
\hline Analfabeto/a & - & - & $7(100)$ & $7(12)$ & - & - & $2(29)$ & $1(14)$ & $4(57)$ \\
\hline Sin graduado en ESO & $5(24)$ & $4(19)$ & $12(57)$ & $21(38)$ & $7(12)$ & & & & \\
\hline \multirow{2}{*}{$\begin{array}{l}\text { Con el graduado } \\
\text { o superior }\end{array}$} & $7(25)$ & $10(36)$ & $11(39)$ & $28(50)$ & $\begin{array}{c}- \\
21(38)\end{array}$ & $6(29)$ & $7(33)$ & $3(14)$ & $5(24)$ \\
\hline & & & & & $\begin{array}{r}2(07) \\
28(50)\end{array}$ & $10(36)$ & $6(21)$ & $6(21)$ & $4(14)$ \\
\hline \multicolumn{10}{|l|}{ Ocupación } \\
\hline Amas de casa & $5(15)$ & $6(18)$ & $22(67)$ & $33(59)$ & $1(03)$ & $6(18)$ & $11(33)$ & $7(21)$ & $8(24)$ \\
\hline Empleado & $6(29)$ & $8(38)$ & $7(33)$ & $21(38)$ & $33(59)$ & & & & \\
\hline \multirow[t]{2}{*}{ Estudiante } & $1(50)$ & - & $1(50)$ & $2(04)$ & $\begin{array}{r}1(05) \\
21(38)\end{array}$ & $9(43)$ & $4(19)$ & $3(14)$ & $4(19)$ \\
\hline & & & & & $\begin{array}{c}- \\
2(04)\end{array}$ & $1(50)$ & - & - & $1(50)$ \\
\hline \multicolumn{10}{|l|}{ Clase social } \\
\hline Baja & - & 2(17) & $10(83)$ & $12(21)$ & - & $1(08)$ & $6(50)$ & $3(25)$ & $2(17)$ \\
\hline Media & $6(21)$ & $8(27)$ & $15(52)$ & $29(52)$ & $12(21)$ & & & & \\
\hline \multirow[t]{4}{*}{ Alta } & $6(40)$ & $4(27)$ & $5(33)$ & $15(27)$ & $1(03)$ & $10(35)$ & $5(17)$ & $3(10)$ & $10(35)$ \\
\hline & & & & & $29(52)$ & & & & \\
\hline & & & & & $1(07)$ & $5(33)$ & $4(26)$ & $4(26)$ & $1(07)$ \\
\hline & & & & & $15(27)$ & & & & \\
\hline
\end{tabular}

Nota. Values in parentheses are percentages.

variación (análisis chi-cuadrado) las diferencias entre los síntomas de depresión en las 56 pacientes de sexo femenino de Dubai y las 248 pacientes de Estados Unidos (Beck
1993). Se analizó cada uno de los 21 apartados del BDI para determinar con precisión qué síntomas de depresión presentaban diferencias en función del origen étnico de las 
Tabla II

Análisis de las diferencias entre las variables socio-demográficas y las puntuaciones de depresión en BDI y MMPI-D para un total de 56 pacientes

\begin{tabular}{|c|c|c|c|c|c|c|c|c|}
\hline \multirow[b]{3}{*}{$\begin{array}{l}\text { Variables } \\
\text { demográficas }\end{array}$} & \multicolumn{4}{|c|}{ BDI } & \multicolumn{4}{|c|}{ MMPI-D } \\
\hline & \multicolumn{4}{|c|}{ ANOVA } & \multirow[b]{2}{*}{ Media } & \multirow[b]{2}{*}{$\mathrm{SD}$} & \multicolumn{2}{|c|}{ ANOVA } \\
\hline & Media & $\mathrm{SD}$ & $\mathrm{F}$ & $\mathrm{df}$ & & & $\mathrm{F}$ & $\mathrm{df}$ \\
\hline \multicolumn{9}{|l|}{ Edad } \\
\hline $20-24$ & 30,43 & 17,14 & \multirow{5}{*}{, 27} & \multirow{5}{*}{2,53} & 30,00 & 10,50 & \multirow{5}{*}{1,12} & \multirow{5}{*}{2,53} \\
\hline $25-29$ & 26,82 & 10,25 & & & 28,82 & 6,43 & & \\
\hline $30-34$ & ,849 & & & & ,350 & & & \\
\hline \multirow{2}{*}{$35-40$} & 25,69 & 11,24 & & & 27,69 & 7,66 & & \\
\hline & 28,16 & 11,78 & & & 31,92 & 6,29 & & \\
\hline \multicolumn{9}{|l|}{ Estado civil } \\
\hline Casado/a & 28,21 & 12,17 & \multirow{5}{*}{,49 } & \multirow{5}{*}{2,53} & 30,39 & 7,17 & \multirow{5}{*}{,99 } & \multirow{5}{*}{2,53} \\
\hline Soltero/a & 25,24 & 12,33 & & & 28,35 & 7,65 & & \\
\hline Divorciado/a & ,691 & & & & ,402 & & & \\
\hline \multirow[t]{2}{*}{ Viudo/a } & 29,25 & 10,05 & & & 31,50 & 6,81 & & \\
\hline & 34,50 & 9,19 & & & 37,00 & 5,66 & & \\
\hline \multicolumn{9}{|l|}{ Nivel educativo } \\
\hline Analfabeto & 37,86 & 6,91 & \multirow{4}{*}{3,52} & \multirow{4}{*}{2,53} & 35,43 & 4,96 & \multirow{4}{*}{2,66} & \multirow{4}{*}{2,53} \\
\hline Sin graduación en ESO & 27,52 & 11,35 & & & 30,33 & 7,52 & & \\
\hline Con el graduado &, $037 *$ & & & &, $079 *$ & & & \\
\hline o superior & 25,11 & 12,18 & & & 28,57 & 7,11 & & \\
\hline \multicolumn{9}{|l|}{ Ocupación } \\
\hline Amas de casa & 29,24 & 11,90 & \multirow{4}{*}{,79 } & \multirow{4}{*}{2,53} & 30,55 & 6,59 & \multirow{4}{*}{, 79} & \multirow{4}{*}{2,53} \\
\hline Empleado/a & 25,05 & 11,73 & & & 28,90 & 7,72 & & \\
\hline \multirow[t]{2}{*}{ Estudiante } & ,457 & & & & 457 & & & \\
\hline & 27,50 & 16,26 & & & 35,00 & 15,56 & & \\
\hline Clase social & & & & & & & & \\
\hline Baja & 34,17 & 6,93 & & & 31,08 & 4,94 & & \\
\hline Media & 28,03 & 11,26 & 4,25 & 2,53 & 31,00 & 8,17 & 1,28 & 2,53 \\
\hline Alta &, $019 *$ & & & & 286 & & & \\
\hline & 21,53 & 13,68 & & & 27,53 & 6,74 & & \\
\hline
\end{tabular}

$(*) \mathrm{p}<, 05$.

$(* *) \mathrm{p}<, 01$.

pacientes. En 13 de los 21 apartados del BDI se obtuvieron efectos primarios significativos o muy cerca de ser significativos por razón del origen étnico de las pacientes. Fueron los siguientes atendiendo al orden de la magnitud de las diferencias: autorechazo, sentimiento de castigo, pérdida de apetito, pérdida de peso, cambio en el aspecto corporal, fatiga, pensamientos suicidas, obsesión somática, indecisión, insomnio, autoinculpación, irritabilidad y retraimiento social. Los datos relativos se presentan en la tabla III.

Las comparaciones de pares de resultados de los 13 apartados revelaron lo siguiente:
En nueve apartados (a saber, sentimiento de castigo, pérdida de apetito, pérdida de peso, cambio de aspecto corporal, fatiga, obsesión somática, insomnio, irritabilidad y retraimiento social) las pacientes de Dubai presentaban puntuaciones más altas en los síntomas de depresión que las de sus homólogas de los Estados Unidos. En los apartados de autorechazo, pensamientos de suicidio, indecisión y autoinculpación, las pacientes de Estados Unidos ofrecían puntuaciones más altas que las pacientes de Dubai. Los datos relativos se presentan en la tabla IV. 
Tabla III

Análisis del test chi-cuadrado de los síntomas depresivos y de la etnia (56 pacientes de Dubai y 248 pacientes estadounidenses) en cada uno de los 21 ítems del test BDI

\begin{tabular}{lrccc} 
Items & chi-cuadrado & significado & $\begin{array}{c}\text { Pacientes de Dubai } \\
\% \text { respuesta }\end{array}$ & $\begin{array}{c}\text { Pacientes de USA } \\
\% \text { respuesta }\end{array}$ \\
\hline 1- Tristeza & 2,15 &, 142 & $89 \%$ & $81 \%$ \\
2- Pesimismo &, 76 &, 383 & $77 \%$ & $82 \%$ \\
3- Sentimiento de faracaso &, 55 &, 458 & $73 \%$ & $68 \%$ \\
4- Insatisfacción &, 41 &, 522 & $89 \%$ & $92 \%$ \\
5- Culpabilidad &, 40 &, 526 & $63 \%$ & $67 \%$ \\
6- Castigo & 26,68 &, $0000^{* * *}$ & $71 \%$ & $34 \%$ \\
7- Rechazo de uno mismo & 39,22 &, $0000^{* * *}$ & $63 \%$ & $93 \%$ \\
8- Auto-culpabilidad & 3,91 &, $047^{*}$ & $82 \%$ & $91 \%$ \\
9- Ideas de suicidio & 5,86 &, $015^{* *}$ & $41 \%$ & $59 \%$ \\
10- Llanto & 1,32 &, 250 & $79 \%$ & $71 \%$ \\
11- Irritabilidad & 3,41 &, $065^{*}$ & $93 \%$ & $83 \%$ \\
12- Actitud antisocial & 3,35 &, $067^{*}$ & $86 \%$ & $74 \%$ \\
13- Indecisión & 4,66 &, $031^{* *}$ & $61 \%$ & $75 \%$ \\
14- Cambio de imagen física & 9,67 &, $001^{* *}$ & $79 \%$ & $56 \%$ \\
15- Dificultad para trabajar & 1,08 &, 296 & $80 \%$ & $86 \%$ \\
16- Insomnio & 3,92 &, $047^{*}$ & $84 \%$ & $71 \%$ \\
17- Fatiga & 6,95 &, $008^{* *}$ & $95 \%$ & $80 \%$ \\
18- Pérdida del apetito & 22,93 &, $0000^{* * *}$ & $79 \%$ & $43 \%$ \\
19- Pérdida de peso & 11,67 &, $0006^{* * *}$ & $43 \%$ & $21 \%$ \\
20- Preocupación somática & 5,74 &, $017^{* *}$ & $70 \%$ & $52 \%$ \\
21- Pérdida de la líbido &, 99 &, 320 & $66 \%$ & $59 \%$ \\
\hline
\end{tabular}

$(*) \mathrm{p}<, 05$.

$(* *) \mathrm{p}<, 01$.

$(* * *) \mathrm{p}<, 001$.

Tabla IV

Comparación intercultural de los resultados de los 13 ítems del BDI entre los pacientes de Dubai y los norteamericanos

Síntomas depresivos en los que los pacientes

Síntomas depresivos en los que los pacientes de Dubai obtuvieron mejores puntuaciones estadounidenses obtuvieron mejores puntuaciones
1. Sentimiento de sentirse castigado/a
2. Pérdida del apetito
3. Pérdida de peso
4. Cambio de imagen personal
5. Fatiga
6. Preocupación somática
7. Insomnio
8. Irritabilidad
9. Actitud antisocial

1. Rechazo a sí mismo

2. Ideas suicidas

3. Indecisión

\section{Discusión}

Esta serie de pacientes sólo puede considerarse representativa de la depresión clíni- ca presente en los Servicios de Psiquiatría de Dubai. Existen, por supuesto, problemas en la utilización del conjunto de criterios para el diagnóstico de la depresión. 
Los datos sociodemográficos indican claramente que existe una significativa diferencia en el nivel de depresión de las pacientes sin alfabetización en comparación con las pacientes alfabetizadas. Las pacientes sin alfabetización tendían a mostrar más síntomas de depresión grave que las pacientes con alfabetización. Esto puede ser debido al hecho de que las pacientes con alfabetización disponen de mayores oportunidades para afrontar las dificultades y para expresar sus sentimientos de formas diferentes y poseen una mejor percepción acerca del comportamiento apropiado. Esta diferencia puede ser que también refleje las dificultades asociadas a la comprensión de las preguntas, si bien se realizó un esfuerzo para minimizar esta circunstancia. Esto también puede reflejar pautas de búsqueda de ayuda así como la posibilidad de que sea la gravedad de sus síntomas, más que su nivel educativo, lo que conduzca a la búsqueda de ayuda. Pueden mostrarse más reticentes a acudir a los servicios psiquiátricos por el estigma que conlleva, pero no existe evidencia ni en la literatura ni en las costumbres locales que demuestre que esto sea así. Puesto que también es mayor la probabilidad de que pertenezcan a la clase socioeconómica baja, ello puede confirmar los hallazgos obtenidos con anterioridad (Cochrane y Stopes-Roe 1980, Surtees et al. 1983, Dilling y Weyerer 1984, y Madianos et al. 1985).

Los resultados también indican que las pacientes de clase socioeconómica baja mostraban una diferencia significativa mayor en el nivel de depresión que los otros dos grupos. Esto, nuevamente, puede estar en función de la marginación y estatus social. Existen límites en el número y en la clase de alternativas de comportamiento al alcance de los individuos que integran la clase baja y esto puede incrementar una aparente sensación de indefensión e incom- petencia. Además, puede ser que los integrantes de las clases media y alta estén preparados para la experiencia de la depresión gracias al mayor nivel de educación y socialización. Esperamos estudiar los papeles y expectativas individuales de los sujetos atendiendo a su propio papel y a las expectativas de los demás hacia el individuo. Lo haremos en el estudio principal que seguirá a la investigación piloto.

Nuestros resultados también confirman que las manifestaciones sintomáticas de depresión varían entre culturas. El perfil sintomático de depresión entre las pacientes de Dubai era un tanto diferente al de las pacientes de Estados Unidos. Las pacientes de Dubai obtuvieron puntuaciones significativamente más altas que las pacientes de Estados Unidos en los apartados del BDI de sentirse castigadas, pérdida de apetito, pérdida de peso, cambio de aspecto corporal, fatiga, obsesión somática, insomnio, irritabilidad y retraimiento social e inferiores que las pacientes de Estados Unidos en los apartados de autorechazo, pensamientos suicidas, indecisión y autoinculpación. Sería preciso que dicha comparación se pusiera en correlación con el entorno religioso y la actividad religiosa. Es necesario hacer una llamada de atención con respecto a la comparación de datos entre culturas en periodos de tiempo diferentes, así como respecto a la utilización de categorías "étnicas". Sin embargo, parece ser que nuestra muestra en Dubai tendría más probabilidades de reflejar una asociación entre síntomas de la depresión y factores orgánicos o somáticos, lo que es coherente con los valores culturales. Así la expresión de síntomas físicos puede ser más aceptable culturalmente que la expresión de problemas emocionales. Además, las mujeres de Dubai disponen de menos posibilidades de hablar acerca de sus problemas psicológicos para no atraer la 
atención de los demás sobre cómo se sienten y por miedo a perjudicar la reputación de sus familias y llevar el deshonor a las mismas. Un número significativo de nuestras pacientes habían sido enviadas desde otros departamentos médicos al Departamento de Psiquiatría, porque no eran consideradas como "realmente enfermas".

Cuando los síntomas del BDI de Dubai se compararon con los síntomas en Estados Unidos surgieron interesantes diferencias interculturales en la presentación de los síntomas. Teniendo presentes los problemas señalados con anterioridad, en cuanto a que la utilización de "mecanismos étnicos" condujeran al establecimiento de una categoría engañosa (como es definida por Kleinman 1987) parece que en nuestra muestra la autodisciplina y la autoinculpación son inferiores como ocurre con el intento de suicidio. De este modo, en una cultura en la que la noción de uno mismo es atribuida no al individuo sino a su papel en la familia y en la sociedad (véase Soffan 1986) no es sorprendente que la autoinculpación sea inferior. Resultaría interesante establecer la correlación existente entre esto con las nociones y la explicación del individuo acerca del locus interno o externo del control de su enfermedad. De nuevo, los niveles elevados de obsesión somática no resultan sorprendentes. La fatiga y la irritabilidad presentan una incidencia alta en otros estudios procedentes de culturas no occidentales (e.g., Avasthi et al. 1991, Mahatme et al. 1989). Puede que el MMPI no sea la herramienta de investigación más adecuada, pero la derivación de categorías de personalidad (en la actualidad en estudio) nos permitirá analizar la personalidad premórbida y la disposición.

Aún es preciso añadir una considerable labor de estudio, incluyendo investigaciones cualitativas en detalle acerca de cómo son percibidos los síntomas de la depresión en las culturas de Oriente Medio. Este estudio piloto ha conducido a un vasto análisis cualitativo, que se encuentra en marcha actualmente, acerca de cómo es percibida la depresión por la cultura de los Emiratos Árabes Unidos. Al mismo seguirá un estudio encaminado a estimar la frecuencia de los síntomas de la depresión entre las mujeres de Dubai en grupos diferentes y culturalmente diversos (nativas, árabes, asiáticas, europeas), evaluando cómo intervienen estas variaciones interculturales en la forma en que se manifiestan los síntomas depresivos y estudiando si existen diferencias en la interpretación de la depresión por parte de la población en general y por parte de la población psiquiátrica.

\section{Conclusiones}

Este estudio preliminar es el primero de los estudios realizados sobre la depresión de las mujeres de Dubai que fija su atención en los síntomas del trastorno.

Este estudio parece indicar que sería de utilidad para los profesionales de la salud mental en los EAU, el que fueran conscientes de las diferencias en la sintomatología depresiva según los grupos étnicos, así como de los factores culturales y sociales que influyen en la manifestación de los síntomas en las impresiones sobre la enfermedad que las pacientes explican al psiquiatra $\mathrm{y}$ en su posterior respuesta al tratamiento.

Los resultados aportados en el presente estudio representan más un punto de partida que la concreción de una teoría definitiva acerca de la distribución de los síntomas depresivos entre las mujeres en Dubai y en otros entornos culturalmente diversos. 


\section{Bibliografía}

AL-HAAJ, F.M.A. Depression. $1^{\text {st }}$ ed., Islamic Library, Beirut, 1981.

ASHURST, P., HALL, Z. Understanding Women in Distress. Routledge, London, 1991.

AVASTHI, A., KHAN, M.K.R., ELROEY, A.M. Inpatient Sociodemographic and Diagnostic Study from a Psychiatric Hospital in Libya. Int. J. of Social Psychiat., vol. 37, n. ${ }^{\circ} 4$, pp. 267-279, 1991.

BEBBINGTON, P.E. The Epidemiology of Depressive Disorder. Cult. Med. and Psychiat. vol. 2, pp. 297-346, 1978.

BECK, A.T. et al. Cognitive Therapy of Depression. Guilford Press, New York, 1979.

BECK, A.T., STEER, R.A. Beck Depression Inventory, Manual. The Psychological Corporation, USA, 1993.

COCHRANE, R., STOPES-ROE, M. Factors Affecting the Distribution of Psychological Symptoms in Urban Areas of England. Acta. Psychiat. Scand., vol. 61, 445-460, 1980.

DILLING, H., WEYERER, S. Prevalence of Mental Disorders in the Small-Town-Rural Region of Trauntein. Acta. Psychiat. Scand., vol. 69, 60-79, 1984.

GHAREEB, G.A. Standardisation of Beck Depression Inventory (BDI) in UAE Society. Social Affairs J., vol. 27, 43-60, 1990.

GHAREEB, G.A. The Nature of Adolescent Depression in UAE. The Egyptian J. of Mental Health, vol. 33, 45-67, 1992.

GHUBASH, R.O.S. Socio-Cultural Change and Psychiatric Disorder: An Epidemiological Study of Women in the Emirate of Dubai (UAE). Unpublished Ph.D. thesis, University of London, 1992.

HATHAWAY, S.R., McKINLEY, J.C. Manual for Administration and Scoring: MMPI-2 Minnesota Multihasic Personality Inventory-2. University of Minnesota Press Minneapolis, Minnesota, 1989.

KLEINMAN, A., GOOD, B. Culture and Depression: Studies in the Anthropology and Cross-Cultural Psychiatry of Affect and Disorder. University of California Press, Los Angeles, 1985.

KLEINMAN, A. Social Origins of Distress and Disease: Depression, Neurasthenia and Pain in Modern China. Yale University Press, London, 1986.

KRAUSE, N., LIANG, J. Cross-Cultural Variations in Depressive Symptoms in Later Life. Int. Psychiat., vol. 4(2), 185-202, 1992.
LEHTINEN, V., JOUKAMAA, M. Epidemiology of Depression: Prevalence, Risk factors and Treatment Situation. Acta Psychiat. Scand., 377, 50-58, 1994.

LUSTMAN, P.J. et al. Similarity of Depression in Diabetic and Psychiatric Patients. Psychosomatic Med., 54, 602-611, 1992.

MADIANOS, M., VLACHONIKOLIS, I., MADIANOU, D., STEFANIS, C. Prevalence of Psychiatric Disorders in the Athens Area. Acta Psychiat. Scand., 377, 50-58, 1985.

MAHATME, S.S., DHAVALE, H.S., PATKAR, A.P. Study of Correlation of Intensity of Symptoms with Stressful Life Events in Depressed Patients. Indian. J. Psychiat., Vol. 31(4), 288-292, 1989.

MARSELLA, A. The Measurement of Depressive Experience and Disorder Across Cultures. In Marsella, A. Hirschfeld, R., Katz, M. (Eds.), The Measurement of Depression. Guilford Press, 1987.

MIND, National Association for Mental Health. Stress on Women: Policy Paper on Women and Mental Health. National Association for Mental Health, London, 1992.

OLFSON, M., KLERMAN, G.L. Depressive Symptoms and Mental Health Service Utilisation in a Community Sample. Soc. Psychiatry \& Psychiat. Epidemiol., vol. 27, 161-167, 1992.

PAYKEL, E.S. Life Events, Social Support and Depression. Acta Psychiat. Scand., 377, 50-58, 1994.

SOFFAN, L.U. The Women of the United Arab Emirates. Croom Helm, London, 1986.

SURTEES, P.G., DEAN, C., INGHAM, J.G., KREITMAN, N.B., MILLER, P., Mc C., SASHIDHARAN, S.P. Psychiatric Disorder in Women from an Edinburgh Community: Association with Demographic Factors. British J. of Psychiat., 142, 238-246, 1983.

WEISMAN, M.M. et al. Depression and its Treatment in US Urban Community - 1975-1976. Arch. Gen. Psychiat., vol. 38, 417-421. Page 1 of references, 1981.

Dirección para correspondencia:

Dr. Dinesh Bhugra

Instituto de Psiquiatría

De Crespigny Park

London SE5 8AF

REINO UNIDO 